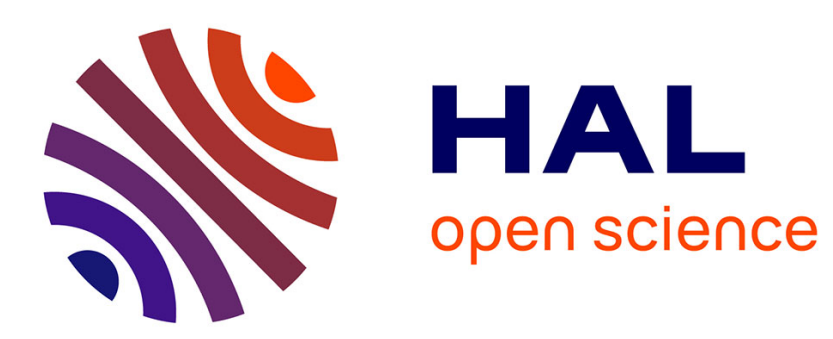

\title{
Domain decomposition approach for nonsmooth discrete problems, example of a tensegrity structure
}

\author{
Shéhérazade Nineb, Pierre Alart, David Dureisseix
}

\section{To cite this version:}

Shéhérazade Nineb, Pierre Alart, David Dureisseix. Domain decomposition approach for nonsmooth discrete problems, example of a tensegrity structure. Computers \& Structures, 2007, 85 (9), pp.499511. 10.1016/j.compstruc.2006.08.027 . hal-00141164

\section{HAL Id: hal-00141164 \\ https://hal.science/hal-00141164}

Submitted on 8 Nov 2016

HAL is a multi-disciplinary open access archive for the deposit and dissemination of scientific research documents, whether they are published or not. The documents may come from teaching and research institutions in France or abroad, or from public or private research centers.
L'archive ouverte pluridisciplinaire HAL, est destinée au dépôt et à la diffusion de documents scientifiques de niveau recherche, publiés ou non, émanant des établissements d'enseignement et de recherche français ou étrangers, des laboratoires publics ou privés. 


\title{
Domain decomposition approach for nonsmooth discrete problems, example of a tensegrity structure*
}

\author{
Shéhérazade Nineb† Pierre Alart $\ddagger$ David Dureisseix ${ }^{\S}$
}

\begin{abstract}
Dealing with numerous bodies in interaction, as occurring in granular media, requires larger and larger computational resources. To this end we develop a domain-decomposition-like method suited to discrete systems with diffuse non smoothness. A multiscale enrichment completes the numerical strategy with extra hope to bridge the gap between discrete and continuum models. The equilibrium of a tensegrity structure, closer to the continuous media case, is chosen to test this approach.

This article in its final form was published in Computers \&5 Structures, Volume 85, Issue 9 (High Performance Computing for Computational Mechanics), May 2007, Pages 499-511.

http://www.elsevier.com doi:10.1016/j.cma.2006.02.003
\end{abstract}

keyword LATIN micro/macro, multiscale, multilevel, substructuring, buckling

PACS 45.10.Db, 46.15.Cc, 46.32.+x, 46.70.Lk

\section{Multiscale discrete systems}

For some years large scale discrete systems have been intensively studied in order to understand the behavior of complex systems which are often discrete if considered at a small enough scale. The increase of the available computational resources allows to use new tools to investigate larger and larger problems. The behavior of atom lattices is studied by physicists and chemists using $a b$ initio computations $[1,2]$. Closer to the continuum mechanics, the interactions of numerous complex mechanisms such as dislocations and grain joints can be simulated to recover the plastic continuous behavior of polycrystalline materials $[3,4]$. Discrete systems can provide some qualitative behavior of continuous

\footnotetext{
* This work is supported by the European Project number IST2001-37172 - SICONOS (Simulation and Control of Non Smooth Dynamical systems)

${ }^{\dagger}$ Corresponding author, Laboratoire de Mécanique et Génie Civil, CNRS UMR 5508 UM2, CC048, Place Eugène Bataillon, F-34095 MONTPELLIER CEDEX 5, FRANCE, nineb@lmgc.univ-montp2.fr

¥alart@lmgc.univ-montp2.fr

$\S$ dureisse@lmgc.univ-montp2.fr
} 
systems $[5,6]$. Nevertheless some systems as particulate media and specifically granular materials are difficult to tackle with a continuous approach; only a discrete modeling can provide relevant results $[7,8]$. The continuous and discrete approaches are complementary and may be associated in some studies to focus the computation on localized large deformations $[9,10,11]$. Some naturally discrete structures as trusses may be favorably modeled as a continuous medium via homogenization techniques $[12,13]$. Many of such structures or media are periodic or quasi-periodic.

In continuous mechanics the medium is viewed as a repetitive structure of identical representative elementary volumes (REV) even if such a representative cell is not always easy to define. For granular materials this definition is still an open question because the medium is naturally discrete and the interactions between components are complex (cf Section 2). For discrete structures we can easily distinguish a basic cell [13] or module [14] whose the global structure is the repetition of.

Domain decomposition methods provide a general framework to the multiscale numerical analysis of such structures. Indeed, substructuring appears as a naturally multiscale numerical strategy where each substructure is related to an intermediate scale between the basic components inside the subdomains and the global structure. Moreover multiscale approaches can enrich the substructuring in incorporating either a micro-macro splitting of some variables [15] or even an homogenization procedure [16]. The motivation of the present work is not mainly in parallelism usage due to the fact that the problems on tensegrity structures are not very large in size (in comparison with granular media problems). Nevertheless, designing an efficient nonsmooth solver is interesting, considering that domain decomposition methods have proved their efficiency for linear problems [17]. Moreover, an interesting feature of the proposed multiscale approach is to provide a numerically homogenized behavior of each substructure, which is useful from a mechanical point of view. This work is a first attempt to extend the LATIN (Large Time Increment) [15] micro-macro approach, initially developed for continuous media, to strongly nonsmooth discrete systems.

\section{A discrete system with diffuse non smooth- ness}

If we define discrete systems as a set of nodes and of links between them, the non smoothness may only occur in the constitutive relations of the links. A granular medium with contact and friction between the grains constitutes a typical example of a fully non smooth system for which all the links involve non smooth behavior: unilateral contact and dry friction. Such a system is the main and ultimate motivation of the present numerical study, but it is quite difficult to define rigorously onto it a domain decomposition approach and to proceed to validation tests. Indeed the well adapted modeling of granular media in a large range of behaviors (from quasi static evolutions to highly dynamical flows) is based on a velocity-impulsion formulation $[18,19]$ in a global time stepping integration scheme. In such a situation, a substructuring of a granular medium has to be frequently updated to take into account the changes of the connectivity of the particles. Beyond this technical difficulty the non smooth 
system to solve at each time step is quite specific with the regularizing property of the dynamical formulation. We preferred in a first approach to consider a static problem involving a discrete structure. Such a situation is closer to elastostatic problems initially investigated with domain decomposition methods [17].

Tensegrity systems are innovative strut and cable systems used in Civil Engineering, Figure 1. Their modeling requires to take into account the diffuse non smoothness through the whole structure. Indeed as poetically defined by Fuller [20], they are viewed as "islands of compression in an ocean of tension"; Motro [21] gives a more precise but general definition: "systems in a stable selfstress state including a discontinuous set of compressed components inside a continuum of tensioned components". Consequently the unilateral behavior of the cables only loadable in tension is a dominant feature through the whole structure and confers on large scale tensegrity systems a good status for testing our multiscale non smooth approach. Such a discrete system is quite different from discretized structures issued from a continuous domain. In the microcracked domain considered in [22] the number of degrees of freedom concerned with contact is small, even if this example involves numerous contact interfaces (cracks). In a previous paper [23], the features of some multicontact problems are discussed and the suited strategies are compared.

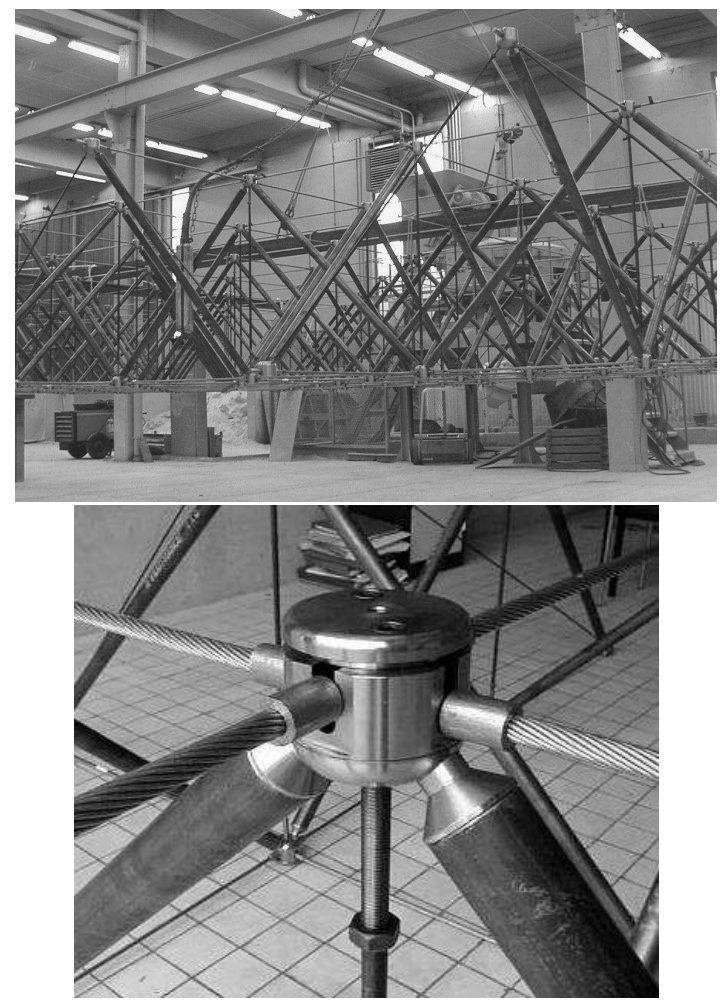

Figure 1: A typical tensegrity structure and a node design, tensarch project (courtesy of R. Motro) 


\begin{tabular}{|l||l|}
\hline$b, c$ & Bar and cable subscripts \\
\hline$t^{0}, t_{b}^{0}, t_{c}^{0}$ & Prestress \\
\hline$e^{0}, e_{b}^{0}, e_{c}^{0}$ & Prestrain \\
\hline$t, t_{b}, t_{c}$ & Additional internal tensions \\
\hline$\tau_{c}=t_{c}^{0}+t_{c}$ & Internal tension in cables \\
\hline$e, e_{b}, e_{c}$ & Length variations \\
\hline$k_{b}, k_{c}$ & Local stiffnesses (in tension for cables) \\
\hline$\lambda_{c}=-e_{c}+k_{c}^{-1} t_{c}$ & Corrected length variations in cables \\
\hline$F, F^{d}$ & Internal and external nodal forces \\
\hline$U, U^{d}$ & Unknown and prescribed nodal displacements \\
\hline$B^{t}$ & Link to node mapping \\
\hline$B$ & Node to link mapping \\
\hline$K_{b}=B_{b}^{t} k_{b} B_{b}$ & Bar network stiffness \\
\hline$K_{c}=B_{c}^{t} k_{c} B_{c}$ & Cable network stiffness \\
\hline$K=K_{b}+K_{c}$ & Global stiffness \\
\hline
\end{tabular}

Table 1: General notations

\section{$2.1 \quad$ Reference problem}

In the set of nodes $\Omega$, we distinguish the subset $\Gamma_{u}$ of the nodes where the displacement is prescribed. Three configurations are to be considered: the current one $\Omega_{1}$ for which the tensions and displacements are unknown, the prestressed configuration $\Omega_{0}$ before applying additional external loading and the relaxed configuration $\Omega_{-1}$ for which the selfstresses are virtually vanished. The three configurations are assumed to be close enough to preserve the principle of small perturbations and the prestresses are assumed to be given. According to Table 1, the main unknowns without superscripts, are the kinematic and static variables from the prestressed configuration to the current one. To exhibit stiffness, tensegrity structures require a prestressing of its components. The configuration $\Omega_{0}$ is the prestressed configuration that we consider as our reference configuration: the internal tensions in all the element are denoted with $t^{0}$.

The balance equation involves the internal and external nodal forces which are related to the total tensions in the bars and cables by the mapping $B^{t}$ :

$$
-F+F^{d}=0
$$

where

$$
F=B^{t}\left(t+t^{0}\right)=\left[\begin{array}{ll}
B_{b}^{t} & B_{c}^{t}
\end{array}\right]\left[\begin{array}{c}
t_{b}+t_{b}^{0} \\
t_{c}+t_{c}^{0}
\end{array}\right]
$$

Note that prestresses $t^{0}$ are self balanced, i.e. $B^{t} t^{0}=0$. The strain admissibility equations connect the displacements of the nodes to the length variations of the bars and cables; the displacements of some nodes are prescribed to clamp the structure to the support:

$$
\begin{gathered}
e=B U=\left[\begin{array}{l}
B_{b} \\
B_{c}
\end{array}\right] U=\left[\begin{array}{l}
e_{b} \\
e_{c}
\end{array}\right] \\
\left.U\right|_{\Gamma_{u}}=U^{d}
\end{gathered}
$$


The constitutive relations differ for the bars and the cables. For the bars we consider a local stiffness between the total, prestress or additional tension and the total, prestrain or current length variation according to the linear law (4).

$$
t_{b}+t_{b}^{0}=k_{b}\left(e_{b}+e_{b}^{0}\right)
$$

The cables involve complementary relations between the total tension $\tau_{c}$ and the corrected length variation $\lambda_{c}$ accounting for the local stiffness of the cables: using the definition of $\tau_{c}$ and $\lambda_{c}$ in (5), the behavior of the cables are given in (6). These last equations can be summarized with the concise notation: $0 \leq \tau_{c} \perp \lambda_{c} \geq 0$.

$$
\begin{aligned}
\tau_{c}=t_{c}+t_{c}^{0} & \text { and } \quad \lambda_{c}=-e_{c}+k_{c}^{-1} t_{c} \\
& \left\{\begin{array}{l}
\tau_{c} \geq 0 \\
\lambda_{c} \geq 0 \\
\left(\tau_{c}\right)^{t} \lambda_{c}=0
\end{array}\right.
\end{aligned}
$$

$e_{b}^{0}$ is the prestrain in bars. With the previously mentioned small perturbations, it is related to the prestress $t_{b}^{0}$ with $e_{b}^{0}=k_{b}^{-1} t_{b}^{0}$.
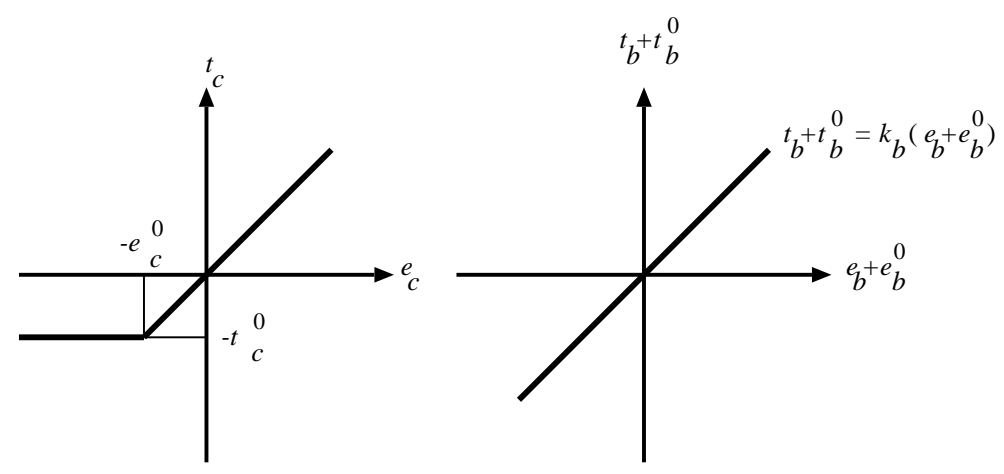

Figure 2: Cable and bar behaviors

These constitutive relations are summarized in Figure 2. Using the balance equation (1), the bar part of the strain admissibility (2) and the bar constitutive law (4) we deduce an equation with $U$ and $\tau_{c}$ as unknowns, related by the bar network stiffness $K_{b}$.

\subsection{Inextensible cables and QP formulation}

If we assume that the cables are inextensible, a quadratic minimization problem (QP) may be postulated where the total cable tension appears as the Lagrange multiplier of the constraint $B_{c} U \leq 0$,

$$
\left\{\begin{array}{l}
K_{b} U+B_{c}^{t} \tau_{c}=F^{d}-B_{b}^{t} t_{b}^{0} \\
0 \leq-B_{c} U \perp \tau_{c} \geq 0
\end{array}\right.
$$

It is equivalent to:

$$
\min _{B_{c} U \leq 0} \frac{1}{2} U^{t} K_{b} U-U^{t} F^{d}-U^{t} B_{b}^{t} t_{b}^{0}
$$


The quadratic function of the QP problem is not necessary lower bounded because the $K_{b}$ matrix is positive but not definite. Indeed the kernel of $K_{b}$ contains the rigid motions of all the bars which are not linked between each other. Therefore a solution, if it does exist, belongs to the edge of the constraint set: either the solution is zero and the structure is perfectly rigid due to the inextensible cables, or at least one cable slackens. Such situations are not realistic because the cable are usually more flexible than the bars. Consequently we have to consider extensible cables (6) in our modeling.

\subsection{Extensible cables and LCP formulation}

Taking into account (1), (2), (3), (4) and the definition of the corrected length variation the balance equation may be expressed with the displacement $U$ and the corrected length variation of cables $\lambda_{c}$,

$$
K U+B_{c}^{t} k_{c} \lambda_{c}=F^{d}
$$

where the matrix $K$ is the total stiffness of the structure, sum of the cable network stiffness (using stiffness in tension) and bar network stiffness. It would be the stiffness of the underlying truss only composed of bars. Having taken account the prescribed displacements, the matrix $K$ is assumed to be invertible. Then the equation (9) can be multiplied by $k_{c} B_{c} K^{-1}$ and the displacement $U$ can be eliminated to provide an equation with the couple $\left(\lambda_{c}, t_{c}\right)$ as unknowns,

$$
\left(k_{c}-k_{c} B_{c} K^{-1} B_{c}^{t} k_{c}\right) \lambda_{c}-t_{c}=-k_{c} B_{c} K^{-1} F^{d}
$$

Denoting with $W$ the matrix $k_{c}-k_{c} B_{c} K^{-1} B_{c}^{t} k_{c}$ and identifying $\tau_{c}$ as the dual variable to $\lambda_{c}$, we recover a classical Linear Complementary Problem (LCP) [24] restricted to the cable network,

$$
\left\{\begin{array}{l}
W \lambda_{c}-\tau_{c}=-k_{c} B_{c} K^{-1} F^{d}-t_{c}^{0} \\
0 \leq \lambda_{c} \perp \tau_{c} \geq 0
\end{array}\right.
$$

This LCP can be solved by a large range of numerical algorithms and we chose herein a Conjugate Projected Gradient method well suited to this type of problem where $W$ is positive but not definite [25]. The LCP is then equivalent to a quadratic minimization problem. Such a conjugate gradient algorithm reveals to be efficient for large scale tensegrity systems, but to get the LCP formulation the large matrix $K$ has to be inverted, which can be expensive indeed impossible and can generate numerical inaccuracies. Therefore a domain decomposition approach seems to be an interesting alternative investigated in the next section. However the resolution of the previous LCP by a Projected Conjugate Gradient algorithm provides a reference solution to test the domain decomposition strategy. Finally, note that it is possible to characterize the kernel of $W$ with respect to the one of $K_{b}$, but this is outside the scope of this paper.

\section{Substructuring strategy}

The first step of the problem reformulation consists of a decomposition of the structure into substructures and interfaces (see Figure 3). Each of these components possesses its own variables and equations. We can proceed in two ways: 
either nodes are distributed among substructures, and interfaces are links joining a substructure to another, or links are distributed among substructures, and interfaces are nodes joining a substructure to another. Only this last case is considered herein (see Figure 3).

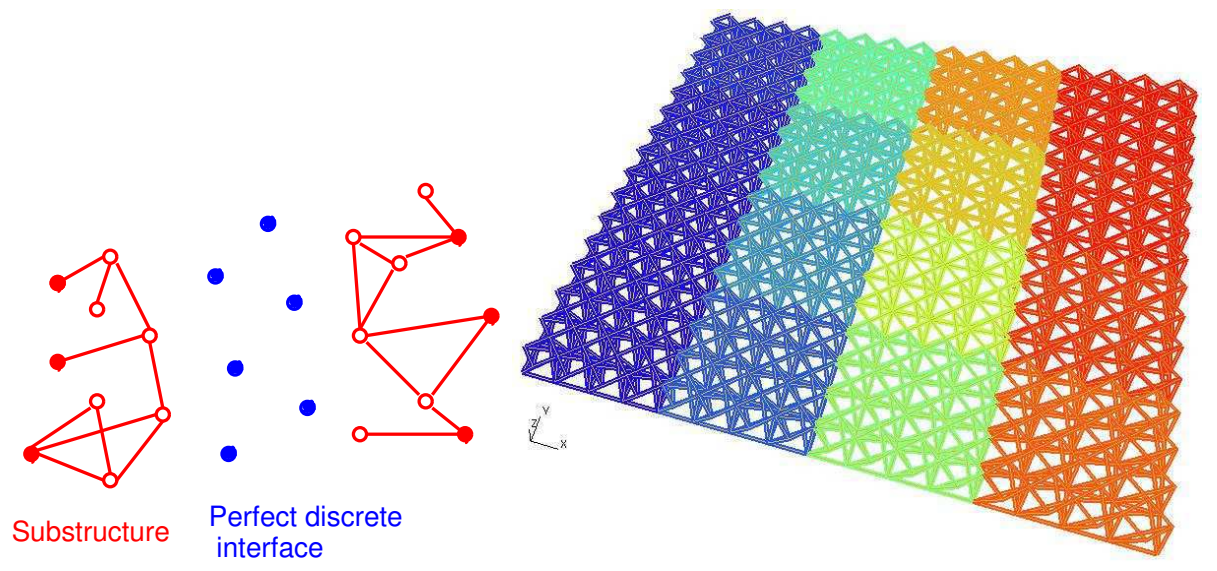

Figure 3: Discrete interface (left) and substructuring of a tensegrity grid (right) with 16 subdomains.

\begin{tabular}{|l||l|}
\hline$F_{E}, F_{E}^{d}$ & Internal and external nodal forces to substructure $E$ \\
\hline$U_{E}, U_{E}^{d}$ & Internal and external nodal displacements to substructure $E$ \\
\hline$\Gamma_{E E^{\prime}}$ & Interface between substructures $E$ and $E^{\prime}$ \\
\hline$\Gamma$ & Global interface \\
\hline$C_{E \Gamma}$ & Boolean mapping matrix of substructure boundary dofs \\
\hline$F_{E \Gamma}$ & Forces of interface $\Gamma$ acting on substructure $E$ \\
\hline$F_{E E^{\prime}}$ & Forces of interface $\Gamma_{E E^{\prime}}$ acting on substructure $E$ \\
\hline$U_{E E^{\prime}}$ & Displacement of $\Gamma_{E E^{\prime}}$ nodes connected to $E$ \\
\hline$U_{E \Gamma}$ & Displacement on substructure $E$ boundary \\
\hline
\end{tabular}

Table 2: Substructuring notations

A substructure $E$ is submitted to the action of its neighboring interfaces $\Gamma_{E E^{\prime}}$ : forces $F_{E E^{\prime}}$ and displacements $U_{E E^{\prime}}$. Extended to all the interfaces local to the substructure $E$, the assembling of the previous fields are denoted with $F_{E \Gamma}$ and $U_{E \Gamma}$. An interface $\Gamma_{E E^{\prime}}$ transfers the forces $F_{E E^{\prime}}$ and the displacements $U_{E E^{\prime}}$ on each of its sides.

The solution $s=\bigcup_{E} s_{E}$ with $s_{E}=\left(e_{E}, t_{E}, U_{E E^{\prime}}, F_{E E^{\prime}}\right)$ of the reference problem must satisfy to:

- the balance equation:

$$
-F_{E}+F_{E}^{d}+C_{E \Gamma}^{t} F_{E \Gamma}=0
$$

where $F_{E}=B_{E}^{t} t_{E}$; 
- the strain admissibility:

$$
\left\{\begin{array}{l}
U_{E \Gamma}=C_{E \Gamma} U_{E} \\
e_{E}=B_{E} U_{E} \\
\left.U_{E}\right|_{\Gamma_{u}}=U_{E}^{d}
\end{array}\right.
$$

- the constitutive relations (4), (6);

- the interface behavior:

force balance:

$$
F_{E E^{\prime}}+F_{E^{\prime} E}=0
$$

continuity of displacements:

$$
U_{E E^{\prime}}=U_{E^{\prime} E}
$$

The interfaces exhibit a perfect behavior because of the continuity of the displacements and because the nonsmoothness is localized within the substructures. This modeling choice is identical to $[26]$ and somehow the dual of the one proposed in [22] where the nonlinearity (contact in crack) is isolated in the interfaces. This substructuring strategy can be easily extended to granular materials where the nodes are the mass centers of the grains and the links are the punctual contacts. But some relations have to be modified according to a velocity-impulse formulation.

\section{A multiscale numerical strategy}

In order to take into account the multiscale aspect of the behavior of a large scale tensegrity structure, especially when it is designed as an assembly of identical modules, a suited numerical strategy has to be settled. A multilevel domain decomposition approach can tackle this task, when the so-called coarse space is related to an homogenized model of the structure [27, 28]. We choose herein to follow the approach proposed in $[15,16]$ that was designed for continuum media, and to extend it to discrete systems.

\begin{tabular}{|l||l|}
\hline$m, M$ & Denote micro and Macro subscripts \\
\hline$l$ & Search direction parameter per substructure \\
\hline$d$ & Search direction parameter per interface \\
\hline$f_{E E^{\prime}}$ & Macro generalized forces of interface $\Gamma_{E E^{\prime}}$ acting on substructure $E$ \\
\hline$f_{E \Gamma}$ & Macro generalized forces of interface $\Gamma$ acting on substructure $E$ \\
\hline$u_{E E^{\prime}}$ & Macro generalized displacements of $\Gamma_{E E^{\prime}}$ nodes connected to $E$ \\
\hline$u_{E \Gamma}$ & Macro generalized displacements on substructure $E$ boundary \\
\hline$R_{E E^{\prime}}^{t}$ & Projector onto generalized macro space \\
\hline
\end{tabular}

Table 3: Micro-macro notations 


\subsection{Micro-macro LATIN method}

\subsubsection{Micro-macro description}

Once a substructuring has been performed, the first step is to describe the media from a microscopic and a macroscopic point of view. Following [28], these descriptions arise from the mechanical fields lying on each interface $\Gamma_{E E^{\prime}}$ independently. Both displacement $U_{E E^{\prime}}$ and forces $F_{E E^{\prime}}$ are split into two parts: the macro part, denoted with a superscript $M$, and the additional micro part, denoted with a superscript $m$. Therefore, one gets

$$
F_{E E^{\prime}}=F_{E E^{\prime}}^{M}+F_{E E^{\prime}}^{m} \quad \text { and } \quad U_{E E^{\prime}}=U_{E E^{\prime}}^{M}+U_{E E^{\prime}}^{m}
$$

The micro and macro spaces must be uniquely defined: they should be 'orthogonal' in a way an orthogonal projector can be used on each subspace. The energy splitting is used in such a way:

$$
\sum_{E E^{\prime}}\left(F_{E E^{\prime}}\right)^{t} U_{E E^{\prime}}=\sum_{E E^{\prime}}\left(F_{E E^{\prime}}^{M}\right)^{t} U_{E E^{\prime}}^{M}+\left(F_{E E^{\prime}}^{m}\right)^{t} U_{E E^{\prime}}^{m}
$$

Superscript $t$ denotes the $L_{2}$ transposition: this energy measure is specific to discrete systems.

\subsubsection{Macro representation}

The macro part lies in a small sized subspace, therefore the macro fields are described with few parameters; a basis of macro fields can be chosen as: $F_{E E^{\prime}}^{M}=$ $R_{E E^{\prime}} f_{E E^{\prime}}$ where $f_{E E^{\prime}}$ stores the macro parameters for forces and $R_{E E^{\prime}}$ is the set of basis vectors. For sake of simplicity, the corresponding macro parameters for the displacement field, $u_{E E^{\prime}}$ is chosen such that the same vector basis holds:

$$
U_{E E^{\prime}}^{M}=R_{E E^{\prime}} u_{E E^{\prime}} \quad \text { and } \quad F_{E E^{\prime}}^{M}=R_{E E^{\prime}} f_{E E^{\prime}}
$$

The macro parameters can be selected depending on the problem one has to model; herein, we choose the generalized averages of fields $F_{E E^{\prime}}$ and $U_{E E^{\prime}}$ on $\Gamma_{E E^{\prime}}$, up to the order 1 . For $3 \mathrm{D}$ analysis, we get:

- for $f_{E E^{\prime}}$, the 3 resultants of forces, the 3 moments, the 2 tensions and the shear in the interface plane, and the dilatation in the interface plane;

- for $u_{E E^{\prime}}$, the corresponding generalized averages are the 3 mean translations, the 3 mean rotations, the 2 stretchings and the 'shearing' in the interface plane, and the expansion in the interface plane.

Therefore, 10 parameters are needed per interface for generalized macro force fields, as well as for the generalized macro displacement fields.

As an additional simplification, the basis $R_{E E^{\prime}}$ is orthonormalized:

$$
R_{E E^{\prime}}^{t} R_{E E^{\prime}}=\mathbf{1}
$$

to get

$$
\left(F_{E E^{\prime}}^{M}\right)^{t} U_{E E^{\prime}}^{M}=f_{E E^{\prime}}^{t} R_{E E^{\prime}}^{t} R_{E E^{\prime}} u_{E E^{\prime}}=f_{E E^{\prime}}^{t} u_{E E^{\prime}}
$$


The consequences when using (17) are the expressions of the orthogonal projector onto the macro space:

$$
f_{E E^{\prime}}=R_{E E^{\prime}}^{t} F_{E E^{\prime}} \quad \text { and } \quad u_{E E^{\prime}}=R_{E E^{\prime}}^{t} U_{E E^{\prime}}
$$

and the orthogonality: $R_{E E^{\prime}}^{t} U_{E E^{\prime}}^{m}=0=R_{E E^{\prime}}^{t} F_{E E^{\prime}}^{m}$.

Furthermore, dealing with discrete systems introduces some specificities:

- together with the expression of energy in (17), discrete systems can lead to weak interfaces (if 3 nodes or less are involved on an interface in 3D, only 9 dofs are concerned; a coarse space with 10 dimensions cannot be defined). In this case, the interface $\Gamma_{E E^{\prime}}$ is not selected as a support for any macro space: $R_{E E^{\prime}}=\varnothing$;

- each interface $\Gamma_{E E^{\prime}}$ is a cloud of nodes and the macro part of a field in $3 \mathrm{D}$ is not trivially defined in this case. Therefore, one needs a general tool to compute $R_{E E^{\prime}}$. The first step is to find the main plane close to the interface. A least square minimization of the distance to the plane is equivalent to find the eigenvectors of the 'inertia' matrix of the set of nodes (each with unitary weight). Once the centroid $G$, and the local basis $\left(n_{1}, n_{2}, n_{3}\right)$ are found, where $n_{3}$ is normal to the interface, see Figure 4, $R_{E E^{\prime}}$ is filled in with the following nodal vectors:

- translations in $n_{i}$ directions,

- rotations along $\left(G, n_{i}\right)$ axis: $n_{i \wedge}(X-G)$, where $X$ is the coordinate vector of the current node,

- extension in $n_{1}$ direction: $n_{1} n_{1}^{t}(X-G)$, extension in $n_{2}$ direction: $n_{2} n_{2}^{t}(X-G)$,

- and finally distortion in $\left(n_{1}, n_{2}\right)$ plane: $\left(n_{1} n_{2}^{t}+n_{2} n_{1}^{t}\right)(X-G)$.

A Gram-Schmidt procedure is then applied to get the orthonormal projector $R_{E E^{\prime}}$.
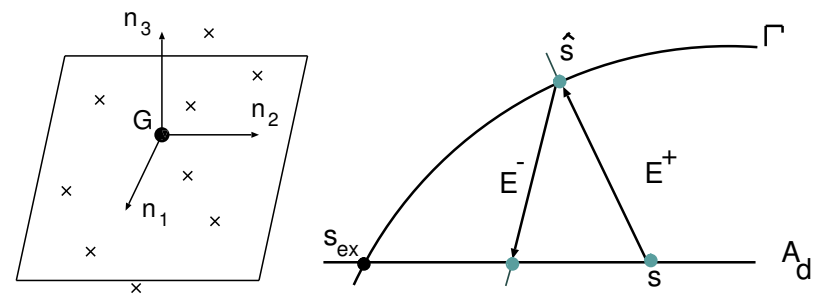

Figure 4: Local basis (left) and LATIN method principles (right)

\subsubsection{LATIN method}

The LATIN method [29] is used as a solver to find the solution of the previous problem. Briefly, it consists of several steps: 
- the equations are split into two sets: (i) the admissible set $\mathbf{A}_{\mathbf{d}}$ with balance equations per substructure (12) and strain compatibility per substructure (13), and (ii) the constitutive relation set $\boldsymbol{\Gamma}$ with link behaviors (14) and interface behavior (15). In the present case, $\boldsymbol{\Gamma}$ is not differentiable due to the diffuse non smoothness of cable behavior;

- an iterative procedure producing alternatively a solution in $\mathbf{A}_{\mathbf{d}}$, and a solution in $\boldsymbol{\Gamma}$, using search directions $\mathbf{E}+$ and $\mathbf{E}-$, Figure 4. The solution of the problem, $s_{\mathrm{ex}}=\left\{\left(e_{E}, t_{E}\right) ;\left(U_{E E^{\prime}}, F_{E E^{\prime}}\right)\right\}$ is the intersection of $\mathbf{A}_{\mathbf{d}}$ and $\boldsymbol{\Gamma}$.

Building $\hat{s}$ once $s$ is known, is the so-called local stage; it involves positive scalar search direction parameters $d$ and $l$ :

$$
\left(\hat{t}_{E}-t_{E}\right)+l\left(\hat{e}_{E}-e_{E}\right)=0
$$

and

$$
\left(\hat{F}_{E E^{\prime}}-F_{E E^{\prime}}\right)-d\left(\hat{U}_{E E^{\prime}}-U_{E E^{\prime}}\right)=0
$$

Building $s$ once $\hat{s}$ is known, is the so-called linear stage: it involves the same parameters as soon as the directions $\mathbf{E}+$ and $\mathbf{E}-$ are conjugate: $\left(t_{E}-\hat{t}_{E}\right)-l\left(e_{E}-\hat{e}_{E}\right)=0$ and $\left(F_{E E^{\prime}}-\hat{F}_{E E^{\prime}}\right)+d\left(U_{E E^{\prime}}-\hat{U}_{E E^{\prime}}\right)=0$.

Such a choice for the search directions ensures the convergence of the method, see [29].

\subsection{Local stage - local non smooth solvers}

As previously mentioned, this stage consists in finding $\hat{s}$ once $s$ is known from the previous stage. It leads to local and linear problems on (perfect) interfaces $\Gamma_{E E^{\prime}}$, and diffuse non smooth local linear complementary problems (LCP) [24] on substructures $\Omega_{E}$.

\subsubsection{For interface $\Gamma_{E E^{\prime}}$}

The behavior (15) is trivially split into micro and macro parts (this is possible thanks to the choice of substructuring we made, that provide perfect interface behavior):

$$
\left\{\begin{array} { l } 
{ \hat { F } _ { E E ^ { \prime } } ^ { M } + \hat { F } _ { E ^ { \prime } E } ^ { M } = 0 } \\
{ \hat { U } _ { E E ^ { \prime } } ^ { M } = \hat { U } _ { E ^ { \prime } E } ^ { M } }
\end{array} \quad \text { and } \quad \left\{\begin{array}{l}
\hat{F}_{E E^{\prime}}^{m}+\hat{F}_{E^{\prime} E}^{m}=0 \\
\hat{U}_{E E^{\prime}}^{m}=\hat{U}_{E^{\prime} E}^{m}
\end{array}\right.\right.
$$

The search direction is also split accordingly with the use of two parameters $d^{m}$ and $d^{M}$ :

$$
\left(\hat{F}_{E E^{\prime}}^{m}-F_{E E^{\prime}}^{m}\right)-d^{m}\left(\hat{U}_{E E^{\prime}}^{m}-U_{E E^{\prime}}^{m}\right)=0
$$

with the same expression for $E^{\prime} E$ fields on the other side of the same interface, and with the corresponding expressions for the macro part, with $M$ superscript.

The solution is explicit:

$$
\left\{\begin{array}{l}
\hat{U}_{E^{\prime} E}^{m}=\hat{U}_{E E^{\prime}}^{m}=\frac{1}{2}\left[\left(U_{E E^{\prime}}^{m}+U_{E^{\prime} E}^{m}\right)-\frac{1}{d^{m}}\left(F_{E E^{\prime}}^{m}+F_{E^{\prime} E}^{m}\right)\right] \\
\hat{F}_{E^{\prime} E}^{m}=-\hat{F}_{E E^{\prime}}^{m}=\frac{1}{2}\left[\left(F_{E^{\prime} E}^{m}-F_{E E^{\prime}}^{m}\right)-d^{m}\left(U_{E^{\prime} E}^{m}-U_{E E^{\prime}}^{m}\right)\right]
\end{array}\right.
$$

A similar expression is obtained for the macro part. 


\subsubsection{For substructure $\Omega_{E}$}

Using a scalar search direction parameter $l$, the problem is independent for cables and bars.

For bars, $\left(\hat{e}_{b}, \hat{t}_{b}\right)$ must verify the constitutive relation (4) and the search direction (20). One gets:

$$
\left\{\begin{array}{l}
\hat{e}_{b}=\left(k_{b}+l\right)^{-1}\left(l e_{b}+t_{b}\right) \\
\hat{t}_{b}=k_{b} \hat{e}_{b}
\end{array}\right.
$$

For cables, $\left(\hat{e}_{c}, \hat{t}_{c}\right)$ must verify the constitutive relation (6). The resulting problem is a LCP [24] once the change of variables (6) is used, to get:

$$
\left\{\begin{array}{l}
\hat{\lambda}_{c}-\left(k_{c}^{-1}+l^{-1}\right) \hat{\tau}_{c}=\tilde{\lambda} \\
0 \leq \hat{\tau}_{c} \perp \hat{\lambda}_{c} \geq 0
\end{array}\right.
$$

where $\tilde{\lambda}=-\left[l^{-1} \tau_{c}+e_{c}+k_{c}^{-1} t_{c}^{0}\right]$ is known at this stage. Its solution is

$$
\left\{\begin{array}{l}
\hat{\lambda}_{c}=<\tilde{\lambda}>_{+} \\
\hat{\tau}_{c}=-\left(k_{c}^{-1}+l^{-1}\right)<\tilde{\lambda}>_{-}
\end{array}\right.
$$

Note that this is the simplest choice for the local stage. Subsequent work will deal with a version where admissibility per substructure is enforced at the local stage, leading to a LCP coupling bars and cables, and for which there exists efficient solvers (cf European Project Siconos, http://siconos.inria.fr). Such a version is under development, in the same spirit as to improve direct linear global solvers with domain decomposition techniques by maintaining them for the smaller problems of independent substructures, and coupling them with iterative procedures on interface only $[17,30]$. Indeed, this would replace global LCP solvers on the whole problem with local solvers per substructure, as the one used in Section 2.

\subsection{Linear stage - linear tangent operator}

This stage is similar to the one described in [16]. Nevertheless, due to the discrete nature of the problem, an algebraic formulation needs to be derived. This is done in this article.

Once $\hat{s}$ is known from the previous stage, the linear stage consists in finding $s$ that satisfies the balance equations and strain admissibility per substructure (12), (13). Considering the macro part, an additional constraint is to enforce macro continuity of the fields at interfaces: with a force-oriented approach, this constraint is to prescribe, on each interface $\Gamma_{E E^{\prime}}$,

$$
F_{E E^{\prime}}^{M}+F_{E^{\prime} E}^{M}=0
$$

To avoid an overconstrained problem, the search direction requires a weak formulation as this is done in the following.

\subsubsection{Macro description and search direction}

First, due to the fact that this stage deals primarily with a substructure-oriented data structure, additional notations are required: the subscript $(E \Gamma)$ will refer 
to the assembly of $\left(E E^{\prime}\right)$ quantities related to the same substructure $E$. For instance, one gets from (18) the column vector related to $\Omega_{E}: F_{E \Gamma}^{M}=R_{E \Gamma} f_{E \Gamma}$.

To manage the additional admissibility of macro space forces (23), these are arising from a single macro field $f_{\Gamma}$ defined on the global interface $\Gamma$ (assembly of local ones $\left.\Gamma_{E E^{\prime}}\right)$. This is truly similar to the Lagrange multiplier used in dual domain decomposition [27]. Therefore, $f_{E \Gamma}=c_{E} f_{\Gamma}$ where $c_{E}$ is a signed boolean matrix, extracting $\left.f_{\Gamma}\right|_{E E^{\prime}}$ or $-\left.f_{\Gamma}\right|_{E^{\prime} E}$ on all interfaces connected to the substructure $\Omega_{E}$. The dual quantity of $f_{\Gamma}$ is obviously $u_{\Gamma}=\sum_{E} c_{E}^{t} u_{E \Gamma}$ : this is the generalized macro displacement jump on interfaces. Due to this additional requirement on macro forces, the weak form of the search direction on interfaces is used:

$$
\begin{aligned}
& \min _{F_{E \Gamma}^{m}, U_{E \Gamma}^{m}, F_{E \Gamma}^{M}, U_{E \Gamma}^{M}} \\
& \frac{1}{2} \sum_{E}\left[\left(F_{E \Gamma}^{m}-\hat{F}_{E \Gamma}^{m}\right)+d^{m}\left(U_{E \Gamma}^{m}-\hat{U}_{E \Gamma}^{m}\right)\right] \frac{1}{d^{m}}\left[\left(F_{E \Gamma}^{m}-\hat{F}_{E \Gamma}^{m}\right)+d^{m}\left(U_{E \Gamma}^{m}-\hat{U}_{E \Gamma}^{m}\right)\right]+ \\
& \frac{1}{2} \sum_{E}\left[\left(F_{E \Gamma}^{M}-\hat{F}_{E \Gamma}^{M}\right)+d^{M}\left(U_{E \Gamma}^{M}-\hat{U}_{E \Gamma}^{M}\right)\right] \frac{1}{d^{M}}\left[\left(F_{E \Gamma}^{M}-\hat{F}_{E \Gamma}^{M}\right)+d^{M}\left(U_{E \Gamma}^{M}-\hat{U}_{E \Gamma}^{M}\right)\right]
\end{aligned}
$$

for generalized macro forces satisfying $F_{E \Gamma}^{M}=R_{E \Gamma} c_{E} f_{\Gamma}$. This produces the strong form of the micro search direction

$$
\left(F_{E \Gamma}^{m}-\hat{F}_{E \Gamma}^{m}\right)+d^{m}\left(U_{E \Gamma}^{m}-\hat{U}_{E \Gamma}^{m}\right)=0
$$

and the weak form of the macro search direction:

$$
A^{M}\left(f_{\Gamma}-\hat{f}_{\Gamma}\right)+\left(u_{\Gamma}-\hat{u}_{\Gamma}\right)=0
$$

where $A^{M}=\sum_{E} c_{E}^{t} R_{E \Gamma}^{t} \frac{1}{d^{M}} R_{E \Gamma} c_{E}$.

A remarkable property is that for perfect interfaces, $\hat{u}_{\Gamma}=0$, and if one chooses $d^{M}=\infty, A^{M}=0$ and the macro search direction reduces to $u_{\Gamma}=0$, i.e. the continuity of generalized macro displacements on interfaces. Since this is expected to lead to an optimal version of the method, we choose in all of the following to take $d^{M}=\infty$.

\subsubsection{Basic formulation of the linear stage}

Using the strain compatibility and the search directions for substituting the internal tensions and micro forces, that is:

$$
\begin{array}{r}
t_{E}=\left(\hat{t}_{E}-l \hat{e}_{E}\right)+l B_{E} U_{E} \\
F_{E \Gamma}^{m}=\left(\hat{F}_{E \Gamma}^{m}+d^{m} \hat{U}_{E \Gamma}^{m}\right)-d^{m}\left(U_{E \Gamma}-R_{E \Gamma} u_{E \Gamma}\right)
\end{array}
$$

the balance equations and the macro search direction lead to:

$$
\begin{array}{r}
\left(K_{E}-C_{E \Gamma}^{t} R_{E \Gamma} d^{m} R_{E \Gamma}^{t} C_{E \Gamma}\right) U_{E}-C_{E \Gamma}^{t} R_{E \Gamma} c_{E} f_{\Gamma}=\hat{F}_{E}^{d} \\
\sum_{E} c_{E}^{t} R_{E \Gamma}^{t} C_{E \Gamma} U_{E}=0
\end{array}
$$


where $K_{E}=B_{E}^{t} l B_{E}+C_{E \Gamma}^{t} d^{m} C_{E \Gamma}$, and

$$
\hat{F}_{E}^{d}=F_{E}^{d}-B_{E}^{t}\left(\hat{t}_{E}-l \hat{e}_{E}-t_{E}^{0}\right)+C_{E \Gamma}^{t}\left(\hat{F}_{E \Gamma}^{m}+d^{m} \hat{U}_{E \Gamma}^{m}\right)
$$

This is a coupled problem on $\left(U_{E}, f_{\Gamma}\right)$ whose interpretation is a domain decomposition method with macro displacement gluing between substructures. $f_{\Gamma}$ can be interpreted as the Lagrange multiplier corresponding to this gluing. This formulation has several drawbacks:

- first, though matrix $K_{E}$ is invertible, due to the term arising from micro search direction on the substructure boundary, the overall matrix is not. In fact, its kernel still contains the rigid body motions of the floating substructure since the trace of such a motion of the interfaces of $\Omega_{E}$ fully lies on the macro displacement space. This drawback is inherent to this approach since this kernel does not produce any strain energy. This is again similar to the FETI method [27] where the rigid body motions are used as a macro space;

- second, due to the term $R_{E \Gamma} d^{m} R_{E \Gamma}^{t}$, the overall matrix is no more sparse ( $R_{E \Gamma}$ couples dofs on the boundary). This would lead to a large CPU overhead. To overcome this difficulty, another formulation of the linear stage is proposed in the following.

\subsubsection{Reformulation of the linear stage}

The proposed approach uses a change of variable: $u_{E \Gamma}^{\star}=u_{E \Gamma}+\frac{1}{d^{m}} f_{E \Gamma}$ such that the balance equations are expressed with the couple of unknowns $\left(U_{E}, u_{E \Gamma}^{\star}\right)$ with the requirement that these unknowns are linked by

$$
u_{E \Gamma}=R_{E \Gamma}^{t} U_{E}
$$

This is finally written as:

$$
\left[\begin{array}{cc}
K_{E} & -C_{E \Gamma}^{t} R_{E \Gamma} d^{m} \\
-d^{m} R_{E \Gamma}^{t} C_{E \Gamma} & d^{m} \mathbf{1}_{E}
\end{array}\right]\left[\begin{array}{c}
U_{E} \\
u_{E \Gamma}^{\star}
\end{array}\right]=\left[\begin{array}{c}
\hat{F}_{E}^{d} \\
f_{E \Gamma}
\end{array}\right]
$$

the resulting matrix is now 'arrow-structured'. Adding the macro search direction

$$
\sum_{E} c_{E}^{t} u_{E \Gamma}=0
$$

and recalling that $f_{E \Gamma}=c_{E} f_{\Gamma}$ closes the problem.

\subsubsection{Deriving the macro homogenized problem}

The homogenized part of the substructure $\Omega_{E}$ behavior is numerically obtained by condensation of the previous system on coarse dofs $u_{E \Gamma}^{\star}$ (performed with a prematurely ended factorization of the overall matrix):

$$
K_{E}^{\star} u_{E \Gamma}^{\star}=f_{E \Gamma}+g_{E}
$$

with

$$
K_{E}^{\star}=d^{m} \mathbf{1}_{E}-d^{m} R_{E \Gamma}^{t} C_{E \Gamma} K_{E}^{-1} C_{E \Gamma}^{t} R_{E \Gamma} d^{m}
$$


and where

$$
g_{E}=d^{m} R_{E \Gamma}^{t} C_{E \Gamma} K_{E}^{-1} \hat{F}_{E}^{d}
$$

is given at this stage. $K_{E}$ is invertible, but the same argument as for the previous formulation shows that $K_{E}^{\star}$ is not: its kernel is the set of the macro part of rigid body motions. Nevertheless, a generalized inverse $K_{E}^{+}$of $K_{E}^{\star}$ can be used [31] to get $u_{E \Gamma}^{\star}$, or equivalently $u_{E \Gamma}$ :

$$
u_{E \Gamma}=L_{E} f_{E \Gamma}+K_{E}^{+} g_{E}+R_{E} \alpha_{E}
$$

with $L_{E}=K_{E}^{+}-\frac{1}{d^{m}} \mathbf{1}_{E}$ : this is the homogenized flexibility of the substructure, i.e. of the corresponding group of tensegrity modules. It corresponds to the generalized kinematic $u_{E \Gamma}$ and sthenic $f_{E \Gamma} . R_{E}$ is the kernel of $K_{E}^{\star}$ (the rigid body motions projected on the macro space), and $\alpha_{E}$ are the corresponding amplitudes, still unknown up to now. Of course, for this solution to exist, the solvability condition must be fulfilled:

$$
R_{E}^{t}\left(f_{E \Gamma}+g_{E}\right)=0
$$

Finally, the macro problem consists of (34), (35) and closure (32). It can be written as:

$$
\left[\begin{array}{cc}
\sum_{E} c_{E}^{t} L_{E} c_{E} & N \\
N^{t} & 0
\end{array}\right]\left[\begin{array}{c}
f_{\Gamma} \\
\alpha
\end{array}\right]=-\left[\begin{array}{c}
\sum_{E} c_{E}^{t} K_{E}^{+} g_{E} \\
g
\end{array}\right]
$$

$\sum_{E} c_{E}^{t} L_{E} c_{E}$ is simply the assembly of macro homogenized flexibilities, $\alpha^{t}=$ $\left[\ldots \alpha_{E}^{t} \ldots\right]$ is the vector storing the rigid body motion amplitudes of the substructures, $N=\left[\ldots c_{E}^{t} R_{E} \ldots\right]$ collects the rigid body motion basis of the kernels, and $g^{t}=\left[\ldots g_{E}^{t} R_{E} \ldots\right]$. This macro problem is small in size (10 times the number of interfaces) and it can be factorized once for all and reused at each iteration, as soon as a constant search direction is used.

\subsubsection{Relocalization}

Once the macro problem is solved, $f_{E \Gamma}$ and $\alpha_{E}$ are known for each substructure $\Omega_{E}$, and $u_{E \Gamma}$ can be post-treated with (34). Backward substitution on (31) is performed to recover

$$
U_{E}=K_{E}^{-1}\left(\hat{F}_{E}^{d}+C_{E \Gamma}^{t} R_{E \Gamma} d^{m} u_{E \Gamma}^{\star}\right)
$$

The micro additional part is $U_{E \Gamma}^{m}=U_{E \Gamma}-R_{E \Gamma}^{t} U_{E \Gamma}=U_{E \Gamma}-R_{E \Gamma} u_{E \Gamma}$ and $F_{E \Gamma}^{m}$ is computed with the corresponding search direction (27). Internal length variation of links is $e_{E}=B_{E} U_{E}$ and $t_{E}$ is computed with the corresponding search direction (27).

\section{A first test on a tensegrity system}

\subsection{Problem settings}

We consider as a test case a tensegrity grid obtained with the duplication of a self stressed elementary module [14]. Such a module is composed of 8 nodes, 12 cables and 4 bars, Figure 5 on the left. The characteristic parameters of this 
module are given in Table 4. Prestressing is such that this module is selfstressed, i.e. $B t^{0}=0$. Therefore, any assembly of such modules will be self balanced automatically. The tested tensegrity grid possesses $16 \times 16=256$ modules, it is split into $4 \times 4=16$ substructures (each containing 16 modules) and 24 strong interfaces, Figure 3. As a comparison point of view, a similar substructuring of a continuum media plate would lead to 16 substructures and only 24 interfaces, none of them being 'weak'. As boundary conditions, the lower nodes on two opposite edges are clamped, and a uniform vertical force field $F^{d}=40 \mathrm{~N}$ is prescribed on every node.

\begin{tabular}{|l||l|}
\hline$H=0.5 \mathrm{~m}$ & Module height \\
\hline$L=1 \mathrm{~m}$ & Module length \\
\hline$S_{c}=0.510^{-4} \mathrm{~m}^{2}$ & Cable section \\
\hline$E_{c}=10^{11} \mathrm{~Pa}$ & Cable Young modulus \\
\hline$S_{b}=2.810^{-4} \mathrm{~m}^{2}$ & Bar section \\
\hline$E_{b}=210^{11} \mathrm{~Pa}$ & Bar Young modulus \\
\hline$t_{c}^{0}=2000 \mathrm{~N}$ & Lower cables prestress \\
\hline$t_{c}^{0}=\sqrt{2} \times 2000 \mathrm{~N}$ & Upper cables prestress \\
\hline$t_{c}^{0}=\sqrt{(}\left(1+4 \frac{H^{2}}{L^{2}}\right) \times 2000 \mathrm{~N}$ & Bracing cables prestress \\
\hline$t_{b}^{0}=-\sqrt{\left(5+4 \frac{H^{2}}{L^{2}}\right) \times 2000 \mathrm{~N}}$ & Bar prestress \\
\hline
\end{tabular}

Table 4: Characteristic parameters
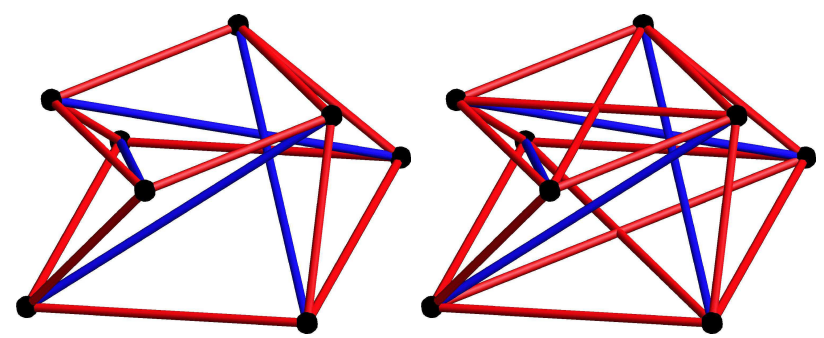

Figure 5: Used modules (initial module on the left and modified module on the right)

\subsection{Choice of search directions}

There are two parameters for this approach that define the search directions: $d^{m}$ and $l$. For the parameter $l$, we used the simplest choice: $l$ is the stiffness of the links of the underlying networks of cables and bars, i.e. $l=k_{c}$ for the cables, and $l=k_{b}$ for the bars. Concerning the parameter $d^{m}$ at the interfaces, a global stiffness of a module has been computed, and the corresponding values of an equivalent stiffness arising from the interfaces alone has been chosen. This procedure has the advantage of being automatic, though the obtained value is not exactly the optimal one. In the present case, it leads to $d^{m}=3.9210^{6} \mathrm{~N} / \mathrm{m}$. For all of the subsequent simulations, these values have been selected once for all, and not been changed in all of the following. 


\subsection{Numerical results}

For the considered test case, the loading level is close to, but less than its ultimate limit value for which there is a lack of stability of the whole structure (similar to global buckling in continuum media and corresponding herein to mechanism occurring).

We consider several loading amplitudes $\alpha F_{d}$ with $0 \leq \alpha \leq 1$. For such values of $\alpha$, the simulation is performed in one step from the reference configuration $\Omega_{0}$ to the current one $\Omega_{\alpha}$, without time stepping. This can be done for the considered problem because it is not an evolution-type one: the final solution does not depend on the loading path.

Figure 6 shows the values of $\tau_{c}$ on each of the 3072 cables, for the different values of $\alpha$, sorted by increasing values. For $\alpha=0$, one recovers the values $\tau_{c}^{0}$ in the cables (cf Table 4). Obviously, as $\alpha$ increases, the stress redistribution is larger and larger: the number of slack cables increases (as well as the maximum value of internal tensions $\tau_{c}$ ) to reach about $14 \%$ of the whole set of cables when $\alpha=1$. For this value of loading, the structure is still within its stable domain for which it still possesses a stiffness reserve. Such simulations are useful to check the integrity of such a structure under extreme loading conditions above normal service usage for which, in general, one assesses that no cable slackens; if this is the case, the strength of the structure could be endangered when the load decreases again and when slacken cables suddenly reload: the rapid change in local apparent stiffness lead to dynamical loadings that can damage the nodes. Figure 7 shows the non linear evolution of this fraction of slack cables when the loading increases, Figure 8 shows the deformed structure (with an amplification coefficient of 10), and the tensions in cables.

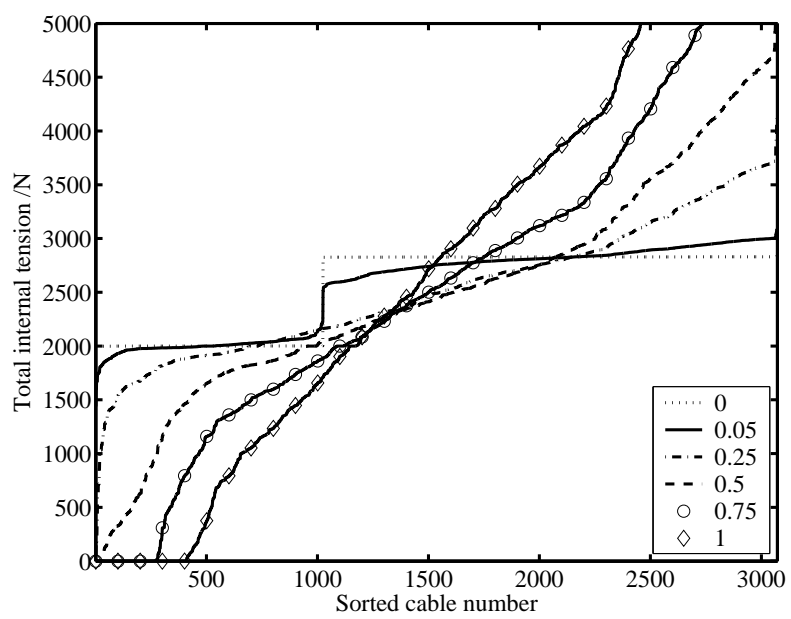

Figure 6: Internal tension in cables for different values of the loading parameter $\alpha$

To test the algorithm convergence, we compute at each iteration an error $e$ with respect to a reference solution obtained as described previously. This error is an energy measure of the distance between the displacement field obtained at 


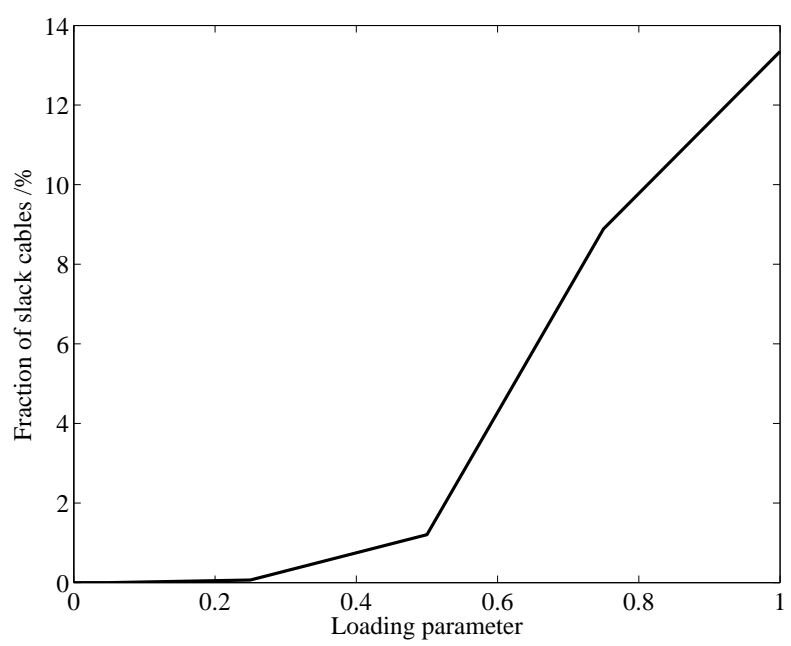

Figure 7: Fraction of slack cables vs loading parameter $\alpha$

the linear stage and the reference displacement field $U_{\text {ref }}$ :

$$
e^{2}=\frac{\frac{1}{2} \sum_{E}\left(B_{E} U_{E}-\left.B_{E} U_{\mathrm{ref}}\right|_{\Omega_{E}}\right)^{t} l\left(B_{E} U_{E}-\left.B_{E} U_{\mathrm{ref}}\right|_{\Omega_{E}}\right)}{\frac{1}{2} \sum_{E}\left(\left.B_{E} U_{\mathrm{ref}}\right|_{\Omega_{E}}\right)^{t} l\left(\left.B_{E} U_{\mathrm{ref}}\right|_{\Omega_{E}}\right)}
$$

Of course, to monitor the algorithm and to stop the iterations, we also use an error indicator $\eta$ that does not require the reference solution. This error indicator is computed after each linear stage, according to a mixed approach; it measures the distance between $\hat{s} \in \boldsymbol{\Gamma}$ and $s \in \mathbf{A}_{\mathbf{d}}: \eta=\frac{\Delta \eta}{\tilde{\eta}}$ with

$$
\begin{aligned}
(\Delta \eta)^{2}=\frac{1}{2} \sum_{\Gamma_{E E^{\prime}}} \Delta F_{E E^{\prime}}^{t} \frac{1}{d^{m}} \Delta F_{E E^{\prime}}+\Delta F_{E^{\prime} E}^{t} \frac{1}{d^{m}} \Delta F_{E^{\prime} E}+ \\
\frac{1}{2} \sum_{E}\left[\left(t_{E}-\hat{t}_{E}\right)+l\left(e_{E}-\hat{e}_{E}\right)\right]^{\prime} l^{-1}\left[\left(t_{E}-\hat{t}_{E}\right)+l\left(e_{E}-\hat{e}_{E}\right)\right]
\end{aligned}
$$

and

$$
\begin{array}{r}
\tilde{\eta}^{2}=\frac{1}{2} \sum_{\Gamma_{E E^{\prime}}} \tilde{U}_{E E^{\prime}}^{t} d^{m} \tilde{U}_{E E^{\prime}}+\tilde{F}_{E E^{\prime}}^{t} \frac{1}{d^{m}} \tilde{F}_{E E^{\prime}}+\tilde{U}_{E^{\prime} E}^{t} d^{m} \tilde{U}_{E^{\prime} E}+\tilde{F}_{E^{\prime} E}^{t} \frac{1}{d^{m}} \tilde{F}_{E^{\prime} E}+ \\
\frac{1}{2} \sum_{E} \frac{\left(e_{E}+\hat{e}_{E}\right)^{t}}{2} l \frac{\left(e_{E}+\hat{e}_{E}\right)^{t}}{2}+\frac{\left(t_{E}+\hat{t}_{E}\right)^{t}}{2} l^{-1} \frac{\left(t_{E}+\hat{t}_{E}\right)^{t}}{2}
\end{array}
$$

where $\Delta F_{E E^{\prime}}=\left(F_{E E^{\prime}}-\hat{F}_{E E^{\prime}}\right)-d^{m}\left(U_{E E^{\prime}}-\hat{U}_{E E^{\prime}}\right), \tilde{U}_{E E^{\prime}}=\frac{1}{2}\left(U_{E E^{\prime}}+\hat{U}_{E E^{\prime}}\right)$, $\tilde{F}_{E E^{\prime}}=\frac{1}{2}\left(F_{E E^{\prime}}+\hat{F}_{E E^{\prime}}\right)$. 


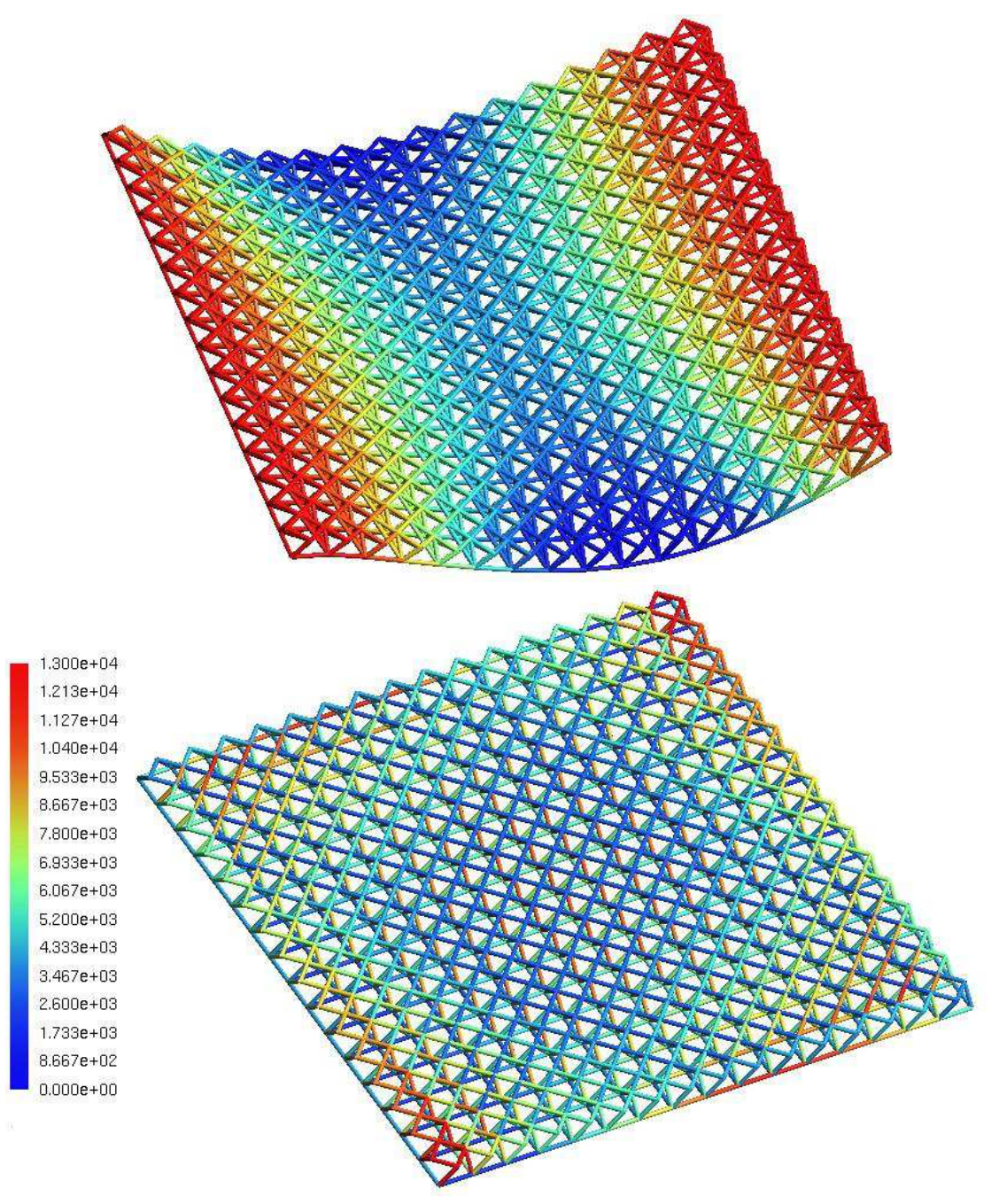

Figure 8: Deformed structure (vertical displacement values) and tensions in cables, for $\alpha=1$ 
Concerning the convergence of the algorithm, Figures 9 and 10 report the evolution of the error $e$ and the error indicator $\eta$ along the iterations, for several values of the loading parameter $\alpha$. For small values of $\alpha$, no cable slackens and the convergence is very similar to the one of the linear case. When $\alpha$ increases, i.e. when the number of slack cables increases, the convergence is affected and soon exhibits two different rates: an initial one, and an asymptotic one for small values of the energy error (here, about $10^{-4}$ ). Such a behavior has to be investigated thoroughly, for instance with a max norm of the error, its projection on the eigenmodes...

Moreover, the proposed approach does not exhibit a constant convergence rate with respect to the non smoothness ratio, defined as the fraction of slack cables of Figure 7. Indeed, when the number of slack cables increases, the tangent stiffness of numerous links can be largely changed. A first improvement of the approach would consist in adapting the search direction $l$ to the local status of cables, but it leads to re-factorizations of the subdomain stiffness matrices, which is a costly part of the algorithm. Doing so only few times during iterations could recover a constant convergence rate along iterations, but probably not along different values of $\alpha$. A second possibility, mentioned above in section 4.2.2 and which is under development, is to use a global search direction per subdomain, adding local admissibility to the local stage problem. In this case, one gets a global LCP problem per subdomain, for which there are some available solvers (cf European Project Siconos, http://siconos.inria.fr).

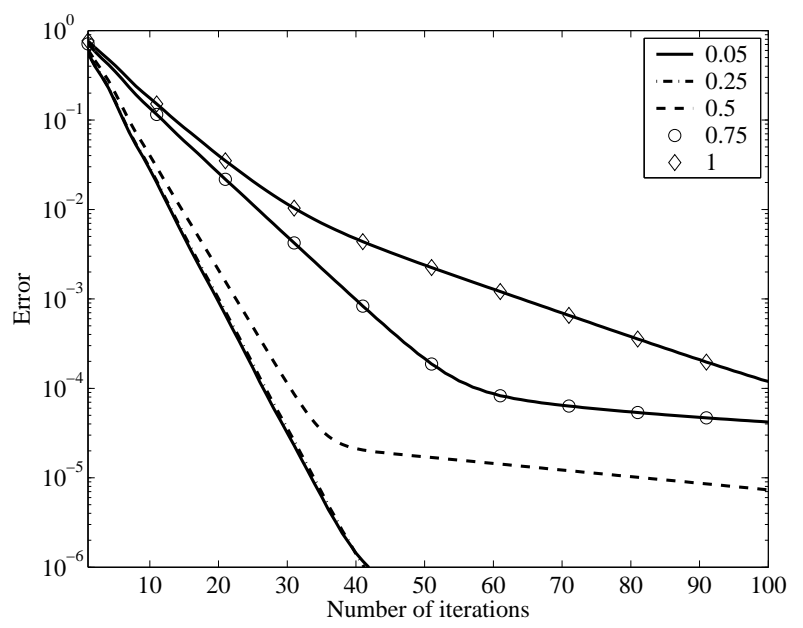

Figure 9: Evolutions of the error with respect to a reference solution, for different values of the loading parameter $\alpha$

\subsection{Comparison with a monodomain approach}

To compare the methods, we use the same algorithm on the same test problem with a unique domain, and therefore without any multiscale feature. In this case, the LATIN approach becomes equivalent to an augmented Lagrangian approach; this is also the case for unilateral conditions arising from frictionless contact [32]. 


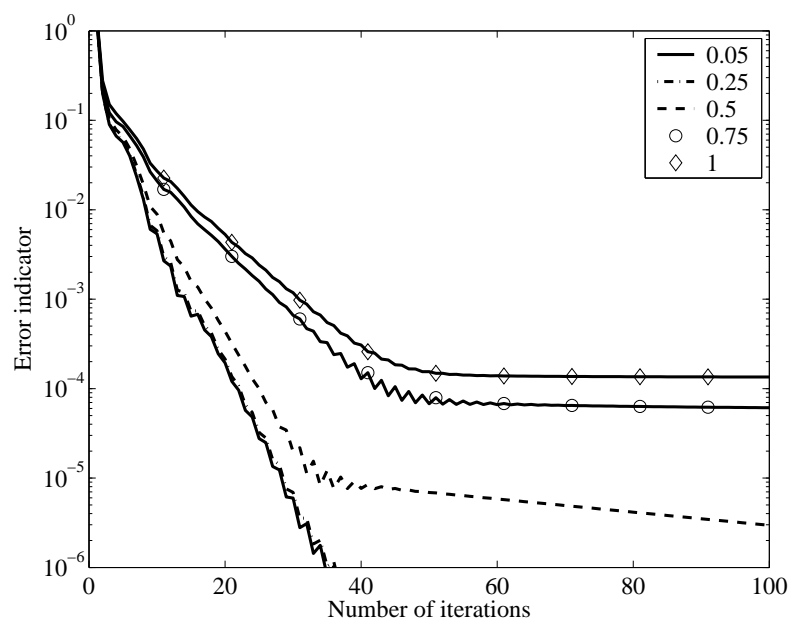

Figure 10: Evolutions of the error indicator, for different values of the loading parameter $\alpha$

The evolution of the error with respect to the number of iterations is shown on Figure 11 for a loading parameter $\alpha=1$. Obviously, the monodomain exhibits a higher convergence rate, which is not surprising if one consider the case of a linear problem, for which the monodomain method is a direct method, while domain decomposition methods require iterations to converge. Therefore, one needs a cost evaluation to compare the two approaches.

Each approach consists of two distinct phases: (i) the initialization phase where the costs are dominated by factorizations of the stiffness matrices, (ii) the iteration phase where, for each iteration, the cost is related on one hand to the local stage for solving the links behaviors, and on the other hand, to solving linear problems (with forward and backward substitutions); during this second phase, the cost is usually dominated by the linear solves and the local stage has the same cost for both approaches.

For the initialization phase, the monodomain approach requires the factorization of the global stiffness matrix, while the multiscale approach requires the factorization of all the local substructure matrices, as well as the macroscopic problem matrix. For the same reasons as the domain decomposition methods outperform direct solvers, the cost of initialization is always higher for the monodomain approach.

For the iteration phase, the cost of one linear solve for the monodomain approach is (in terms of floating point operations) $n l$, where $n$ is the number of dofs, and $l$ is the bandwidth of the global stiffness matrix. Considering the proposed test case, $n=2500$ and $l=200$ (using a reverse Cuthill-McKee renumbering scheme). The number of substructures is $N_{S}=16$, the number of dofs for the local substructure stiffness matrix is $n_{S}=195$ and its bandwith $l_{S}=50$; finally, the number of dofs for the coarse problem is $N=216$ and its bandwidth $L=60$. The ratio of the costs for 1 iteration of the monodomain approach and 
1 iteration of the multiscale approach (on a monoprocessor machine) is

$$
a_{\text {iter }} \approx \frac{n l}{N_{S} n_{S} l_{S}+N L}=2.9
$$

The ratio of convergence rates obtained on Figure 11 to reach an error threshold of $1 \%$ is also close to 3 : during the iteration process, the two algorithms are of the same efficiency. The potential gain therefore lies in the initialization phase, for which the factorization cost ratio is

$$
a_{\text {init }} \approx \frac{\frac{1}{2} n l^{2}}{\frac{1}{2} N_{S} n_{S} l_{S}^{2}+\frac{1}{2} N L^{2}}=11.6
$$

When the problem size increases, if the convergence rate is maintained, we can expect an increase in the efficiency. Indeed, the factorization costs do not increase in the same manner for each approach (typically, $n_{S} \approx n / N_{S}$, and $\left.l_{S} \approx l / \sqrt{N_{S}}\right)$.

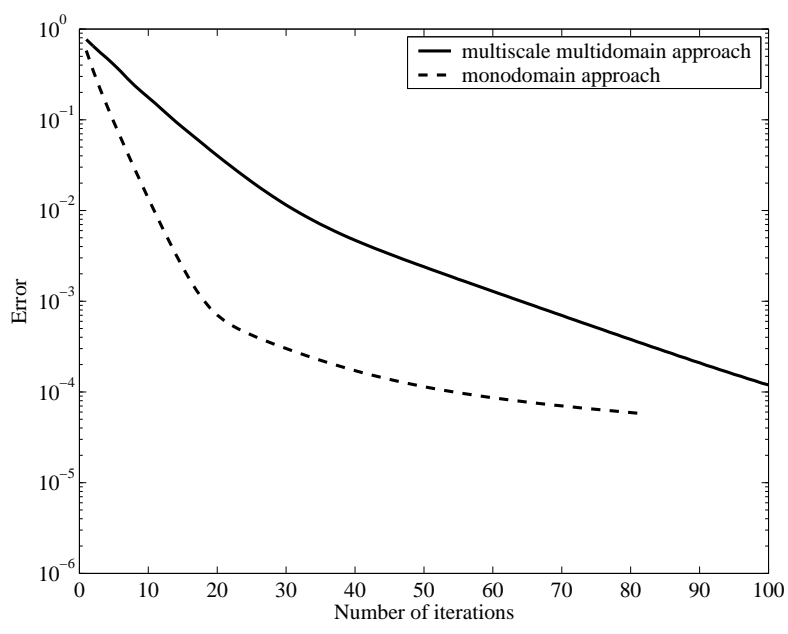

Figure 11: Comparison of error evolutions for different approaches

\subsection{Influence of internal mechanisms}

Finally, one has to note that the module we used in this example exhibits three internal infinitesimal mechanisms [14], and that the assembly of modules in subdomains as well as in the whole structure still possesses one internal mechanism, which is determined only by boundary conditions. To assess the influence of such a mechanism, a modified module is tested; two diagonal crossed cables link the upper four nodes of the module, and two other ones link the four lower nodes, see Figure 5 on the right. In the present case, none of these additional four cables per module are prestressed. Figure 12 shows the error evolution along iterations for each corresponding grid, for $\alpha=1$ and when all the links are modeled as bars (the linear case). The initial convergence rate is improved for the modified modules, though not drastically. 


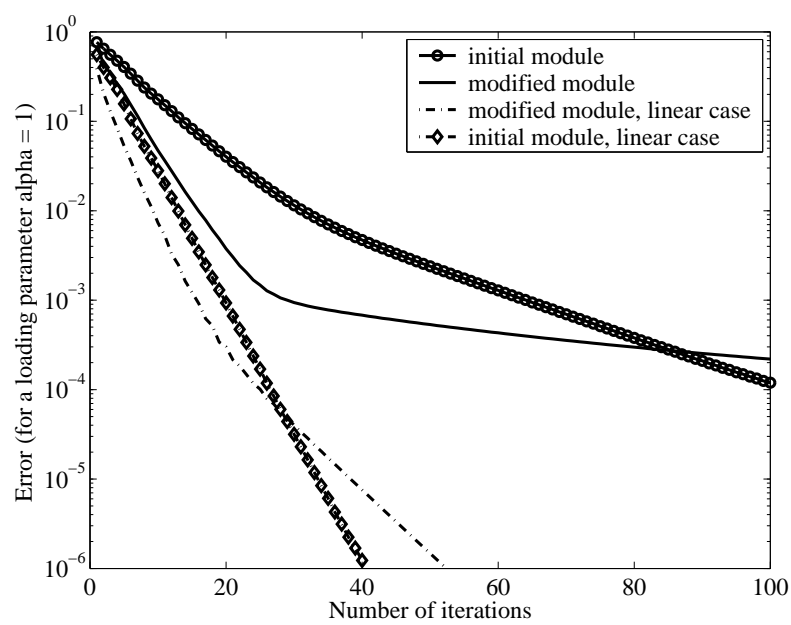

Figure 12: Comparison of error evolutions for different modules

\section{Conclusions and prospects}

The proposed approach is concerned with large scale non smooth problems solved with a substructuring approach. A numerical multiscale algorithm is used, following the one proposed in [16], and extended to discrete systems with diffuse non smoothness. The application is the quasi-static behavior of highly loaded tensegrity structures, when a significant number of cables slacken.

Multilevel domain decomposition algorithms are expected to exhibit numerical scalability with respect to the number of subdomains. This does not pertain with respect to the level of diffuse non smoothness. Adaptations are under work to overcome this difficulty, as proposed in the previous Section.

A first prospect concerns the dynamical behavior of such tensegrity structures. Dynamics could provide a smoother formulation for the problem, but since cables can slack and reload brutally, this may lead to shocks in the solution, and to its associated numerical difficulties.

A second kind of application is the case of granular media simulations where non smoothness occurs with contact and friction interactions between grains $[18,19]$.

Finally, for all of these cases, the algorithm provides also as a second-hand product the generalized (and numerically homogenized) behavior of a substructure, $L_{E}$, that could allow to bridge the gap between the discrete model and the continuum macro model of the structure. This could also lead to a numerical strategy for coupling these different models of the same media in different areas of the structure, when a model adaptation is needed.

\section{References}

[1] F. Cimpoesu, K. Hirao, The ab initio analytical approach of vibronic quantities: Application to inorganic stereochemistry, Advances in Quantum Chemistry 44 (2003) 369-387. 
[2] E. Cancès, M. Defranceschi, W. Kutzelnigg, C. L. Bris, Y. Maday, Computational quantum chemistry: A primer, Hanbook of Numerical Analysis 10 (2003) 3-270.

[3] V. Bulatov, L. Kublin, Dislocation modelling at atomistic and mesoscopic scales, Current Opinion in Solid State and Materials Science 3 (6) (December 1998) 558-561.

[4] C. Lemarchand, B. Devincre, L. Kubin, Homogenization method for a discrete-continuum simulation of dislocation dynamics, Journal of Mechanics and Physics of Solids 49 (9) (2001) 1969-1982.

[5] G. Puglisi, L. Truskinovsky, Mechanics of a discrete chain with bi-stable elements, Journal of the Mechanics and Physics of Solids 48 (1) (January 2000) $1-27$.

[6] O. Kresse, L. Truskinovsky, Mobility of lattice defects: discrete and continuum approaches, Journal of the Mechanics and Physics of Solids 51 (7) (July 2003) 1305-1332.

[7] P. Cundall, O. Stack, A discrete numerical model for granular assemblies, Geotechnique 29 (1) (1979) 47-65.

[8] F. Radjai, D. Wolf, M. Jean, J. Moreau, Bimodal character of stress transmission in granular packings, Phys. Rev. Lett. 80 (1) (1998) 61-64.

[9] J. Fish, W. Chen, Discrete-to-continuum bridging based on multigrid principles, Computer Methods in Applied Mechanics and Engineering 193 (1720) (7 May 2004) 1693-1711.

[10] M. Arroyo, T. Belytschko, A finite deformation membrane based on interatomic potentials for the transverse mechanics of nanotubes, Mechanics of Materials 35 (3-6) (March-June 2003) 193-215.

[11] S. Xiao, T. Belytschko, A bridging domain method for coupling continua with molecular dynamics, Computer Methods in Applied Mechanics and Engineering 193 (17-20) (7 May 2004) 1645-1669.

[12] G. Moreau, D. Caillerie, Continuum modeling of lattice structures in large displacement applications to buckling analysis, Computers and Structures 68 (1-3) (1 July 1998) 181-189.

[13] H. Tollenaere, D. Caillerie, Continuous modeling of lattice structures by homogenization, Advances in Engineering Software 29 (7-9) (11 August 1998) 699-705.

[14] J. Quirant, M. Kazi-Aoual, R. Motro, Designing tensegrity systems: the case of a double layer grid, Engineering Structures 25(9) (2003) 1121-1130.

[15] P. Ladevèze, D. Dureisseix, A micro / macro approach for parallel computing of heterogeneous structures, International Journal for Computational Civil and Structural Engineering 1 (2000) 18-28. 
[16] P. Ladevèze, O. Loiseau, D. Dureisseix, A micro-macro and parallel computational strategy for highly heterogeneous structures, International Journal for Numerical Methods in Engineering 52 (1-2) (2001) 121-138.

[17] P. Le Tallec, Domain decomposition methods in computational mechanics, in: Computational Mechanics Advances, Vol. 1, North-Holland, 1994.

[18] J. J. Moreau, Numerical aspects of sweeping process, Computer Methods in Applied Mechanics and Engineering 177 (1999) 329-349.

[19] M. Jean, The non-smooth contact dynamics method, Computer Methods in Applied Mechanics and Engineering 177 (1999) 235-257.

[20] R. Fuller, The dymaxion world of Buckingham Fuller, 1979.

[21] R. Motro, Tensegrity, Hermes Science Publishing, London, 2003.

[22] A. Nouy, P. Ladevèze, O. Loiseau, A multiscale computational approach for contact problems, Computer Methods in Applied Mechanics and Engineering 191 (2002) 4869-4891.

[23] P. Alart, M. Barboteu, M. Renouf, Parallel computational strategies for multicontact problems: Application to cellular and granular media, International Journal for Multiscale Computational Engineering 1 (2003) 419430 .

[24] Katta, Murty, Principal pivoting methos for LCP, Department of Industrial and Operations Engineering, University of Michigan, 1997.

[25] M. Renouf, P. Alart, Conjugate gradient type algorithms for frictional multi-contact problems: applications to granular materials, Computer Methods in Applied Mechanics and Engineering.

[26] M. Barboteu, P. Alart, M. Vidrascu, A domain decomposition strategy for nonclassical frictional multi-contact problems, Computer Methods in Applied Mechanics and Engineering 190 (2001) 4785-4803.

[27] C. Farhat, F.-X. Roux, A method of finite element tearing and interconnecting and its parallel solution algorithm, International Journal for Numerical Methods in Engineering 32 (1991) 1205-1227.

[28] P. Ladevèze, D. Dureisseix, Une nouvelle stratégie de calcul micro/macro en mécanique des structures, Comptes-Rendus de l'Académie des Sciences 327 (1999) 1237-1244.

[29] P. Ladevèze, Nonlinear Computational Structural Mechanics - New Approaches and Non-Incremental Methods of Calculation, Springer Verlag, 1999.

[30] C. Farhat, F.-X. Roux, Implicit parallel processing in structural mechanics, in: J. T. Oden (Ed.), Computational Mechanics Advances, Vol. 2, NorthHolland, 1994.

[31] C. Farhat, D. Rixen, Encyclopedia of Vibration, Academic Press, 2002, isbn 0-12-227085-1. 
[32] P. Alart, D. Dureisseix, M. Renouf, Using nonsmooth analysis for numerical simulation of contact mechanics, in: Progresses in Nonsmooth Mechanics and Analysis, Kluwer Academic Press, 2005, Ch. 17, to appear. 